\title{
Advanced control of a water supply system: a case study
}

M. Bakker ${ }^{\mathrm{a}, \mathrm{b}}$ (corresponding author), T. Rajewicz ${ }^{\mathrm{c}}$, H. Kien ${ }^{\mathrm{d}}$, J.H.G. Vreeburg ${ }^{\mathrm{e}, \mathrm{f}}$, L.C. Rietveld ${ }^{\mathrm{a}}$

\author{
${ }^{a}$ Faculty of Civil Engineering and Geosciences, Delft University of Technology, P.O. Box 5048, 2600 GA Delft, \\ The Netherlands \\ ${ }^{\mathrm{b}}$ Royal HaskoningDHV B.V., P.O. Box 1132, 3800 BC Amersfoort, the Netherlands (E-mail: \\ martijn.bakker@rhdhv.com, telephone :+31 88348 2327, fax +31 88348 2801) \\ ${ }^{c}$ Aquanet S.A., ul. Dolna Wilda 126, 61- 492 Poznań, Poland \\ ${ }^{\mathrm{d}}$ Oasen N.V., Postbus 122, 2800 AC Gouda, the Netherlands \\ e Sub-Department of Environmental Technology, Wageningen University, P.O. Box 17, 6700 AA Wageningen, \\ The Netherlands \\ ${ }^{\mathrm{f}}$ KWR Watercycle Research Institute, P.O. Box 1072, 4330 BB Nieuwegein, The Netherlands
}

\begin{abstract}
Conventional automatic production flow control and pump pressure control of water supply systems are robust and simple: production flow is controlled based on the level in the clear water reservoir and pump pressure is controlled on a static set-point. Recently, more advanced computerbased control methods were developed in which production flow is controlled by using a shortterm water demand forecasting model and pressure is controlled by a dynamic pressure control module. To assess the differences between conventional and advanced control, we examined operational data of water treatment plant (WTP) Gruszczyn that supplies drinking water to a part of the city of Poznań, Poland. We compared two periods of three weeks of conventional and advanced control. The comparison showed that with advanced control the variation in the production flow was $83 \%$ lower, and the pump pressure of the clear water pumps was $29 \%$ lower. The lower pressure resulted in $20 \%$ less background leakage and the overall system's energy costs were $11.5 \%$ lower.
\end{abstract}

\section{Keywords}

Water demand forecasting; optimal control; pressure management

\section{Introduction}

A water supply system is designed to produce drinking water of good quality, and to supply this water to the consumers under sufficient pressure. The goal in the operation of the system is to produce and supply the drinking water with a high reliability at the lowest operational costs. Initially, the water supply systems were operated manually by operators, but since the mid 1970's water utilities started automating the systems (Bunn, 2007). The control loops were rather straightforward because of the limited computational force of the automation systems. This simple and robust automation is sub-optimal with respect to the performance of the treatment plant and energy efficiency (Bakker et al., 2003). In the meantime, there is an on-going trend towards the fully automated (centralized) operation of water supply systems (Worm et al., 2010; PWN, 2006). 
When utilities implement modern centralized automatic control, they aim at reducing costs and, at the same time, improving the quality of the operations.

A possibility to achieve this goal is to apply a short-term water demand forecasting model for optimal production flow control or optimal pump scheduling. Forecasting models are used by utilities around the world: In the Netherlands in 2012, 57\% of all supplied water was controlled with predictive control models (Bakker et al., 2013a), leading to 5.2\% lower energy costs and 19\% lower turbidity of the clear water. Other examples of the implementation of predictive control are at four large utilities in the United States (Bunn and Reynolds, 2009), where a reduction of energy costs of $12 \%$ was achieved. Another possibility to improve the operation of water supply systems is the implementation of pressure management. In most cases, implementing pressure management includes both creating smaller pressure zones, called district metered areas (DMAs), and installing pressure reducing valves (PRVs) in the distribution network. Pressure management can lead to a reduction of the water losses. Girard and Stewart (2007) showed in their case study a reduction of the water losses of $21 \%$ as a result of reducing the pressure in the DMAs. Pressure management, including the reduction of water losses, also leads to a reduction in the system's energy consumption (Colombo and Karney, 2005).

In this paper, we present a case study of the implementation of advanced control software that aims to combine the two control strategies mentioned above. The software thus controls both the production flow based on an adaptive water demand forecasting model, and the pump pressure by applying flow depending (dynamic) dynamic pressure control.

\section{Material and methods}

\subsection{Case study}

Water company Aquanet S.A. is responsible for the water supply in the city of Poznan (550,000 inhabitants), in the central western part of Poland. Like most water supply companies in Poland, Aquanet manually operated the water treatment and pumping facilities by operators. In 2011 Aquanet decided to fully automate the control of the system supplied by water treatment plant (WTP) Gruszczyn, to run the system unmanned, and to optimize the control of the system, resulting in a better water quality and in a reduction of operational costs by minimizing the energy consumption. The lay-out of the system is shown in Figure 1 and Figure 2. 


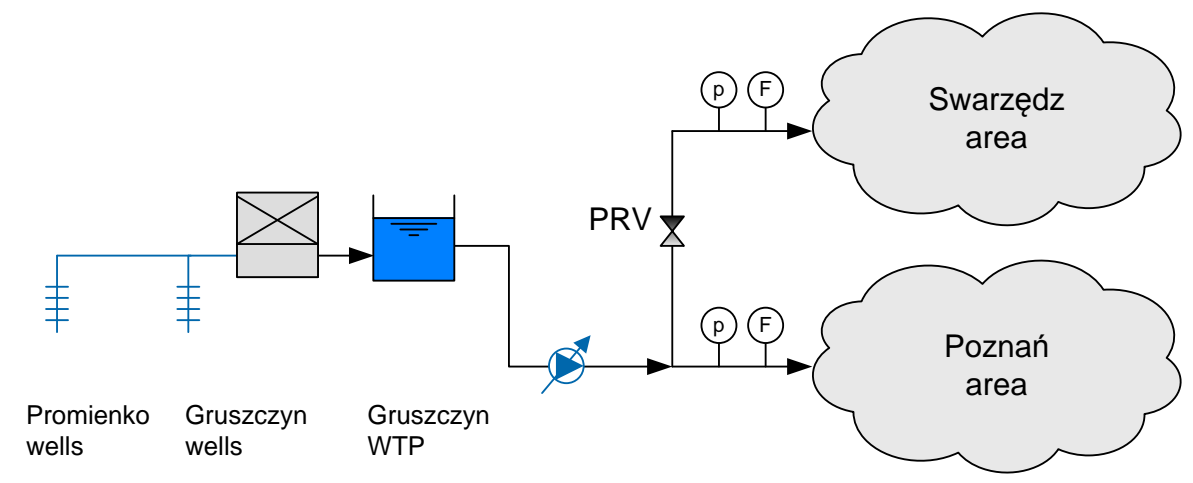

Figure 1: Schematic drawing of the water supply system of Gruszczyn

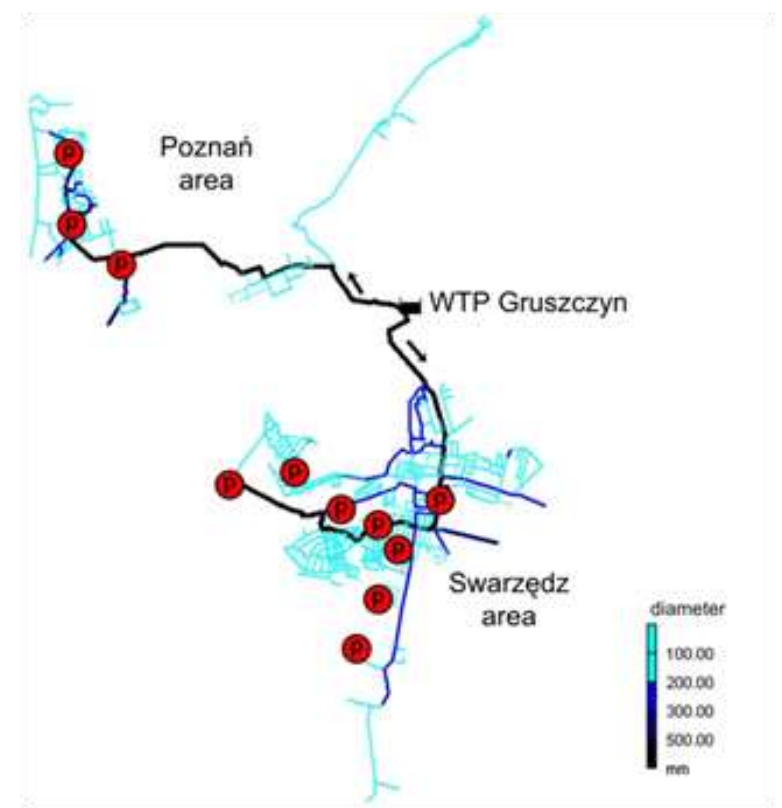

Figure 2: Distribution system of Gruszczyn, including nine new and two existing pressure measuring points

\subsection{Production flow control}

At first, a relatively simple production flow control loop was programmed in the programmable logic controller (PLC). In this control loop the production flow set-point was directly derived from the level in the clear water reservoir. An increasing level resulted in a decreasing set-point, and vice versa. This level based production flow control was capable of controlling the production unmanned. However, many production flow changes occurred, which resulted in energy inefficiency and suboptimal water quality.

In a second stage, a predictive production flow control method was installed. In this control method, the adaptive short-term water demand forecasting model described by Bakker et al. (2013b) was 
used. The forecasting model was installed to forecast the water demand in both supply areas of the system (see Figure 1), and the sum of both was the forecast of the total outflow from the clear water reservoir. The predictive production flow control method calculates the optimal inflow into the reservoir, being as constant as possible. The production control method executes the following calculations to derive the desired inflow set-point:

1. The forecasted total outflow from the clear water reservoir is derived from the forecasted water demands in both areas.

2. This forecasted outflow is transformed into two cumulative series, where:

a. The first series is increased by the buffer volume between the actual level and the maximum allowed level.

b. The second series is decreased by the buffer volume between the actual level and the minimum allowed level.

3. The possible production flows are transformed to cumulative series as well (the method always calculates with discrete flows; if the production flow can be controlled continuously, the method internally calculates with small discrete flow steps);

4. The intersection point of a possible production flow with the minimum / maximum allowed levels depicts the time frame that the production flow can be kept constant without violating the allowed levels.

Figure 3 shows the forecasted outflow (upper graph), the transformation to the minimum and maximum allowed levels, and the evaluation of the possible production flows (lower graph). The production flow control method selects the flow that has the longest timeframe in which the allowed levels are not violated. With this selected flow, the water can be produced with a minimum of flow changes. 

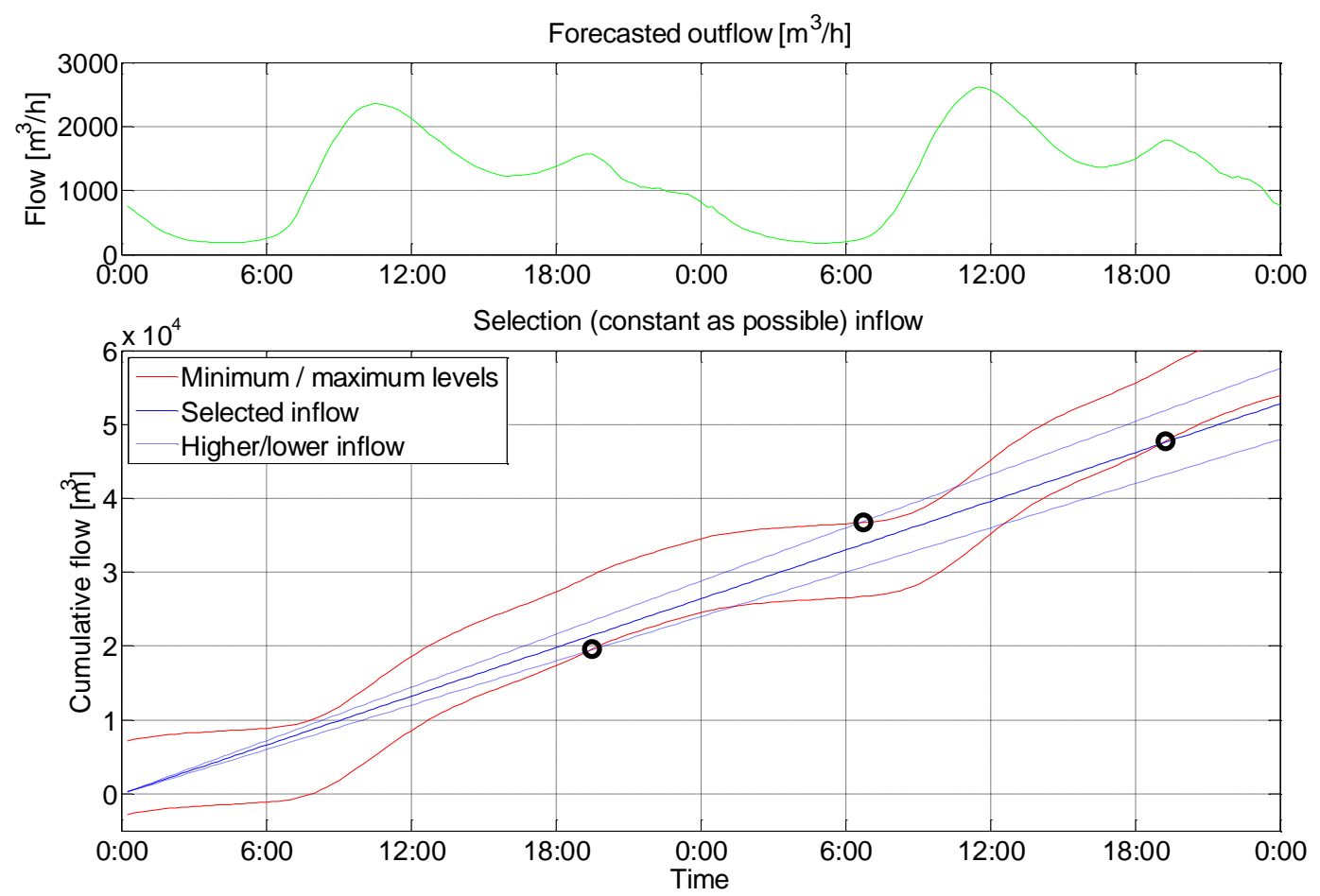

Figure 3: Example of the selection of the desired production flow by transforming the forecasted outflow to minimum / maximum reservoir levels and evaluating discrete production flows

\section{Influence of production flow control}

Production flow control influences the variability of the production flow, which can be expressed in the production variation per day $\left(P V_{d}\right)$. The production variation is defined as the sum of (the absolute values of) the difference between subsequent hourly average production flow values $\left(Q_{\text {prod,d,h}}\right)$ divided by the total daily production:

$$
P V_{d}=\frac{\sum_{h=1}^{h=24}\left|Q_{\text {prod, }, h}-Q_{\text {prod }, d, h-1}\right|}{\sum_{h=1}^{h=24} Q_{\text {prod }, d, h}} \cdot 100 \%
$$

Production flow control also influences the energy consumption for the production of the drinking water. Because real-time energy measurements were not available, the energy consumption for abstraction and treatment $\left(P_{\text {prod }}\right)$ was estimated with:

$$
P_{\text {prod }}=P_{\text {base }}+C_{1} \cdot Q_{\text {prod }}+C_{2} \cdot Q_{\text {prod }}^{3} \quad[k W]
$$

Where $P_{\text {base }}[\mathrm{kW}]$ is the constant, flow independent, energy consumption, $Q_{\text {prod }}\left[\mathrm{m}^{3} / \mathrm{h}\right]$ is the production flow, $C_{1}$ is a value representing energy consumption for static head loss and $C_{2}$ for dynamic head loss in the abstraction and treatment process. We estimated the values for $P_{\text {base }}, C_{l}$ and $C_{2}$ in such a way that the calculated yearly energy consumption matched with the total energy 
consumption that could be derived from the energy bill of 2011. The specific energy consumption for abstraction and treatment was $0.456 \mathrm{kWh} / \mathrm{m}^{3}$.

\subsection{Pressure control}

The distribution pumping station of WTP Gruszczyn consisted of five identical pumps all equipped with variable speed drives (VSD). The pumps were operated as one group at a fixed pump pressure. The clear water was pumped in two directions towards the individual supply areas. Initially the two areas were separated by a PRV in order to reduce the pressure in one zone while keeping a higher pressure in the other. The installed PRV was a medium driven automatic control valve Cla-val NGE9001 (DN250). The operators chose a relatively high pressure set-point for the clear water pumping station, because information about the pressure in the entire network during flow variations was lacking.

In the automation and optimization process, nine new pressure measuring points were installed in the distribution network (see Figure 2). The measurements showed that there was no need to separate the two pressure zones, and that the existing PRV could be removed. After removing the PRV, the pressures in both zones were equalized. For the control of the clear water pumping station, a dynamic pressure control module (DPCM) was installed. In the conventional control loop, the pressure set-point was a static value chosen by the operator. The DPCM is a feedback control model, which dynamically calculates a pressure set-point for the pumping station by comparing the measured pressures at the measuring points with their individual lower bound values. The measuring point with the lowest pressure in relation to its lower bound value is the master in the control loop. The DPCM uses a proportional integral derivative (PID) control mechanism to derive a pressure set-point for the pumping station, based on the desired (lower bound) and measured pressure value of this master pressure measuring point. Figure 4 shows the user interface of the DPCM. 


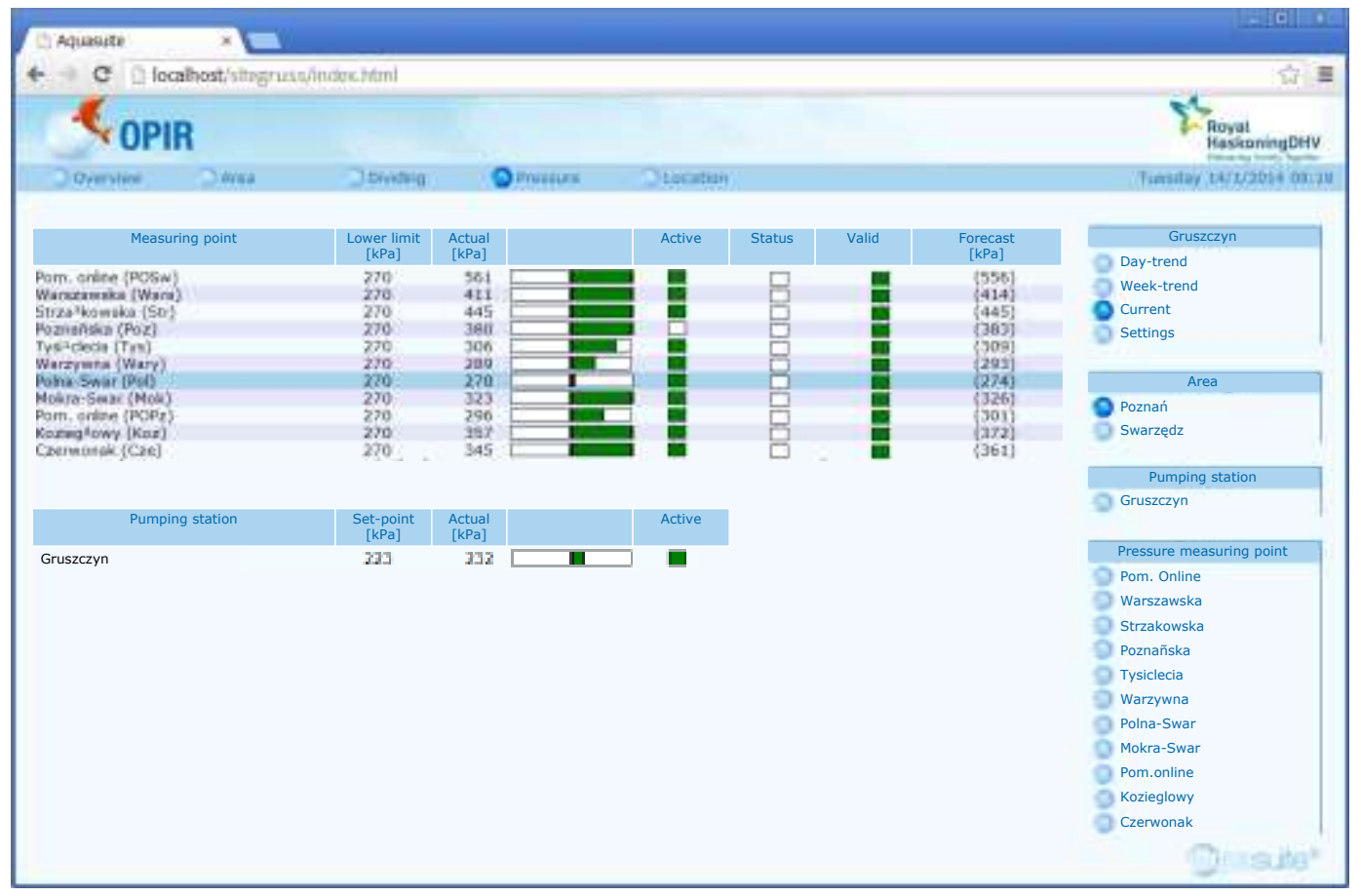

Figure 4: Interface of the dynamic pressure control module (DPCM), showing all measured pressures and their lower bound values, and highlighting which measuring point is the master in the control loop

\section{Using off-line pressure measuring points}

The nine installed pressure measuring points were equipped with a local logger and GSM modem. The measured pressures were not available in real-time, but stored locally and sent to the SCADA system of WTP Gruszczyn once per day. However, the DPCM estimated the real-time pressure $p_{i}$ for each pressure measuring point $i$ as a function of the real-time measured pressure at the pumping station $p_{p s}$ and distribution flow to the area $Q_{d i s t}$, with:

$$
p_{i}=p_{p s}+a+b \cdot Q_{d i s t}^{2}[k P a]
$$

The values for $a$ and $b$ in equation (3) were derived by the DPCM using the least-squares method on the data of the previous 72 hours (see Figure 5). With this functionality, the DPCM is a feedback control model that uses a predicted value as input, and can therefore be qualified to be a hybrid form of a predictive controller and a feedback controller as described by Ulanicki et al. (2000). 


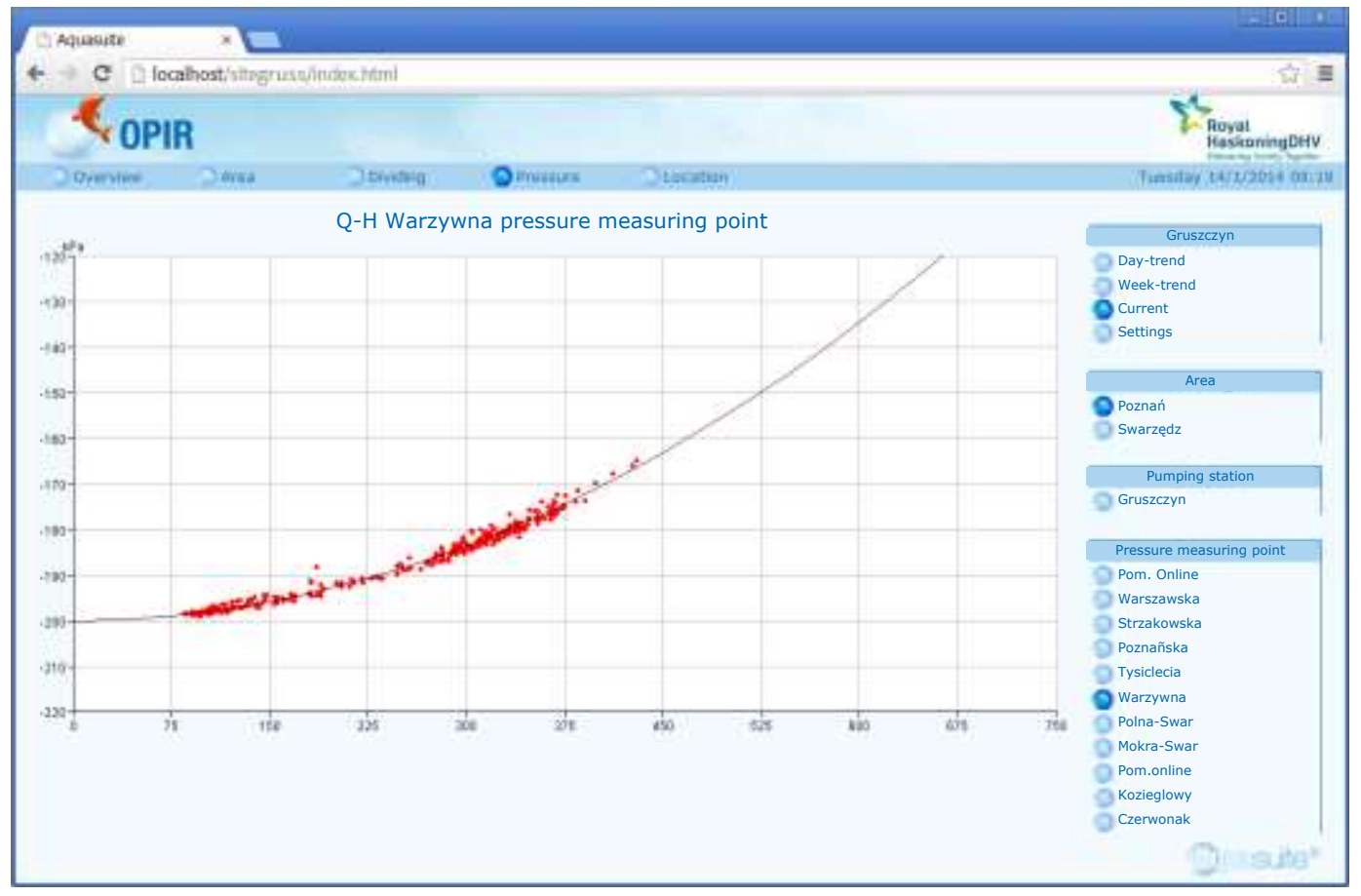

Figure 5: Least squares fit of measured pressure drop between pumping station and pressure measuring point as a relation of the flow to the area

\section{Influence of pressure control}

Pressure control influences the average pressure in the water distribution network and as a result also the leakage in the distribution network. The background leakage $Q_{\text {leak }}$ can be described by (Gomes et al., 2011; Araujo et al., 2006; Vairavamoorthy and Lumbers, 1998):

$$
Q_{\text {leak }}=K_{f} \cdot p^{\beta}\left[m^{3} / h\right]
$$

Where $K_{f}$ is a leakage coefficient for the area, $p$ is the average pressure in the area, and $\beta$ is pressure exponent. According to Gomes et al. (2011), the pressure component $\beta$ varies between 0.5 (for leaks with a fixed leaking area, which is the case for steel pipes or other rigid pipes) and 2.5 (for leaks with a leaking area which is highly sensitive for pressure fluctuations, which is the case for HDPE pipes or other flexible pipes). Gomes et al. (2011) use a value of 1.0, but Ulanicki et al. (2000) use a value of 1.5 for background leakages. As proposed by May (1994) and adapted by Araujo et al. (2006) we will use 1.18 for $\beta$ in this paper.

If the pressure in the area changes, the background leakage in the area will change according to:

$$
\frac{Q_{\text {leak }, 1}}{Q_{\text {leak }, 2}}=\left(\frac{p_{1}}{p_{2}}\right)^{\beta}
$$


A reduction of the leakage will lead to a reduction in the amount of water to be pumped, treated and abstracted. Therefore, also the energy consumption will be reduced. This reduction of energy consumption $\left(d E_{\text {loss }}\right)$ can be estimated by:

$$
d E_{\text {loss }}=d V_{\text {loss }} \cdot E_{\text {spec,tot }}[k W h]
$$

Where $d V_{\text {loss }}$ is the difference in water loss in the water distribution system and $E_{\text {spec,tot }}$ is the total specific energy consumption for abstraction, treatment and distribution, which was $0.600 \mathrm{kWh} / \mathrm{m}^{3}$ (Aquanet, 2012).

Changing the pump pressure affects the energy consumption by the clear water pumps. This difference in energy consumption $\left(d E_{\text {pump }}\right)$ is calculated by:

$$
d E_{\text {pump }}=\frac{\rho \cdot V \cdot d p}{1000 \cdot 3600 \cdot \eta} \quad[k W h]
$$

Where $\rho$ is the specific mass of water $\left(1,000 \mathrm{~kg} / \mathrm{m}^{3}\right), V$ is the total pumped volume of water, $d p$ is the difference in pump pressure, and $\eta$ is the total efficiency of pump, motor plus VSD of the clear water pumps (estimated to be constant at 0.69 as observed by Hydratek (2013)).

\section{Results}

\section{Comparison of operational periods}

To evaluate the results of the improvements in control, the operational data (flows, pressures, water levels) of a period with conventional control were compared to a period of optimized automatic control. The implementation of the advanced control software was done in several phases, and after initial implementation a period of tuning followed. Therefore, a contiguous period with a sharp transition from conventional control to advanced control was not available. Afterwards, we compared, for both control strategies, a three weeks period in November 2011 and 2012 for conventional and control, respectively.

\section{Production flow control}

Figure 6 shows trends of the total water demand, production flow and reservoir level of both examined periods. 

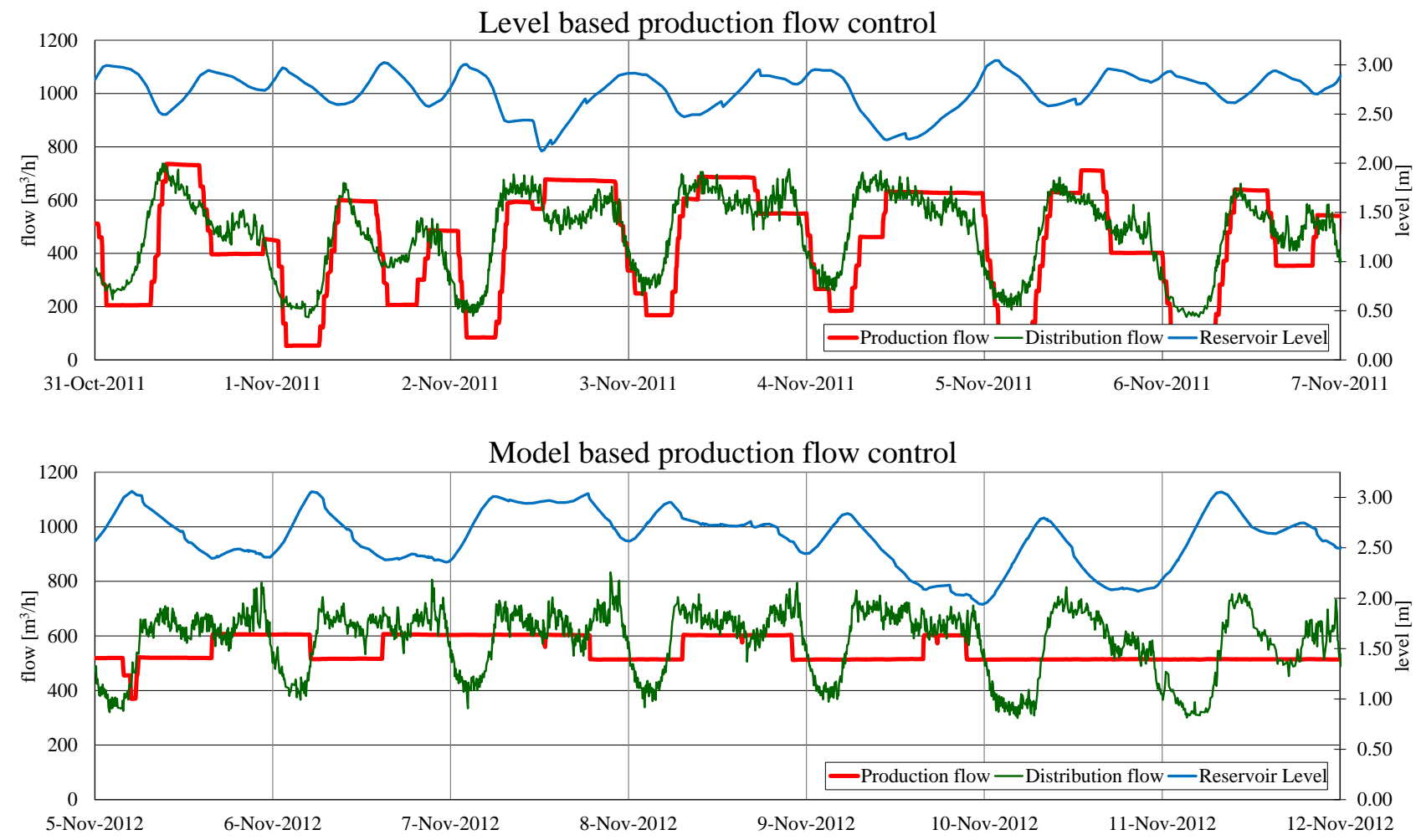

Figure 6: Difference between level based (upper graph) and model based (lower graph) production flow control

The trend with level based control shows that the production flow was switched up and down every day, and that the minimum and maximum flows were almost equal to the minimum and maximum distribution flows. The trend with model based control shows a more stable production flow, with a smaller difference between maximum and minimum flows. Table 1 shows that model based control led to a $83 \%$ lower value for the production variation calculated with equation (1). Bakker et al. (2013a) showed that treatment performance was better at lower values of production variation, resulting in lower values of the turbidity of the clear water. Table 1 also shows that the difference between the maximum and minimum production flows was $67 \%$ lower with model based control. A third aspect shown in Table 1 is the energy consumption and energy costs, which were calculated with equation (2) for both periods. With predictive control, the energy consumption [in $\mathrm{kWh} / \mathrm{m}^{3}$ ] was $1.9 \%$ lower, and relatively more energy was consumed during low tariff hours resulting in $2.7 \%$ lower energy costs. 
Table 1: Difference between level based and model based production flow control

\begin{tabular}{|l|c|c|c|}
\hline & Level based & Model based & Difference \\
\hline Production Variation [\%] & 9.3 & 1.6 & $-83 \%$ \\
\hline Production flow & 52 & 367 & $+600 \%$ \\
Min. flow $\left[\mathrm{m}^{3} / \mathrm{h}\right]$ & 875 & 636 & $-27 \%$ \\
Max. flow $\left[\mathrm{m}^{3} / \mathrm{h}\right]$ & 823 & 269 & $-67 \%$ \\
Max.-Min. $\left[\mathrm{m}^{3} / \mathrm{h}\right]$ & & & \\
\hline Energy $(\mathrm{est})$. & 0.456 & 0.447 & $-1.9 \%$ \\
Cons. $\left[\mathrm{kWh} / \mathrm{m}^{3}\right]$ & 27.43 & 26.68 & $-2.7 \%$ \\
Cost $\left[€ / 1,000 \mathrm{~m}^{3}\right]$ & \multicolumn{2}{|}{} \\
\hline
\end{tabular}

\section{Pressure control}

Figure 6 and Figure 7 show trends of the water demand, the outlet pressure at the pumping station and the average pressure in the area of both examined periods.
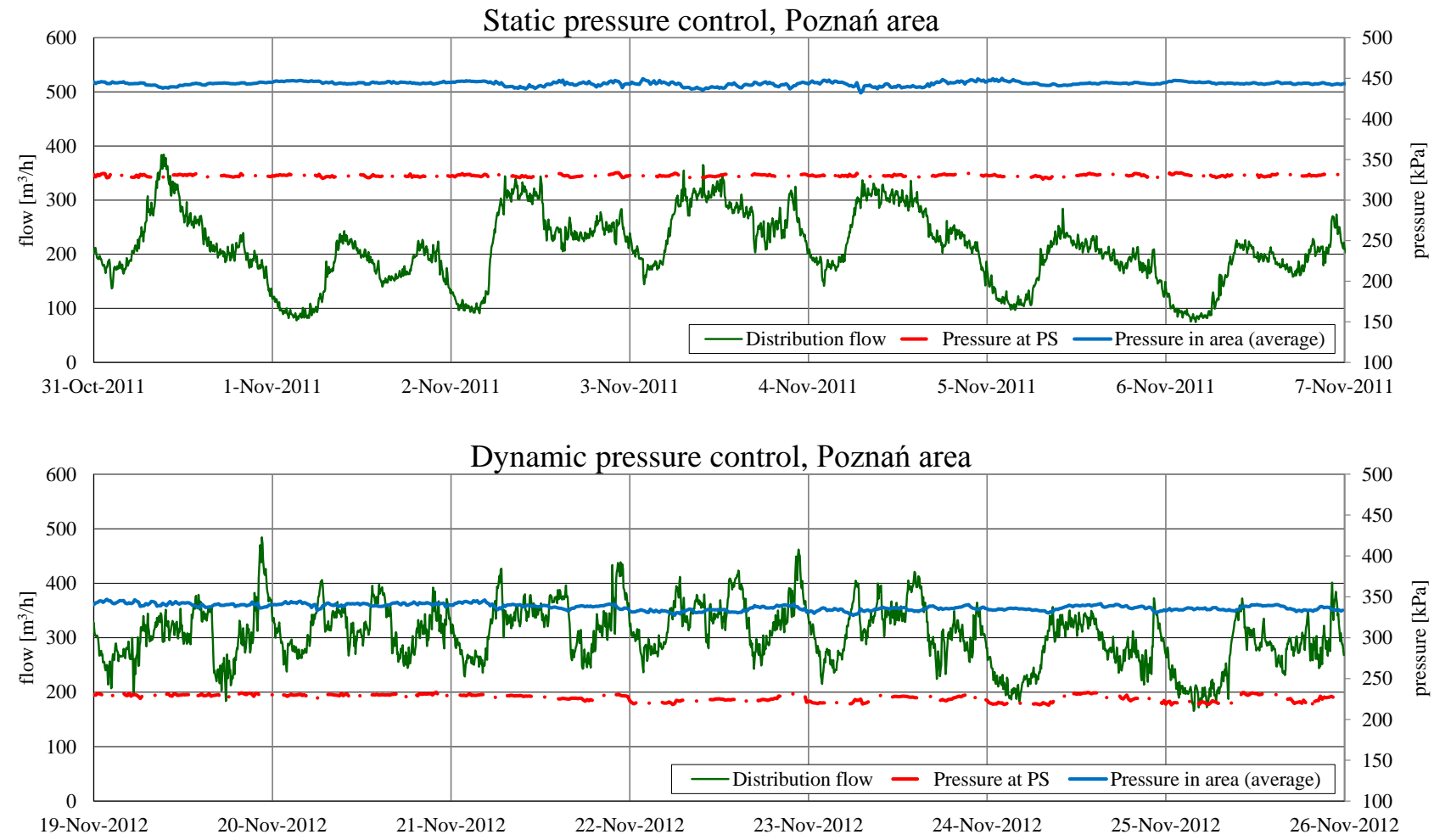

Figure 7: Difference between static (upper graph) and dynamic (lower graph) pressure control,

Poznań area 

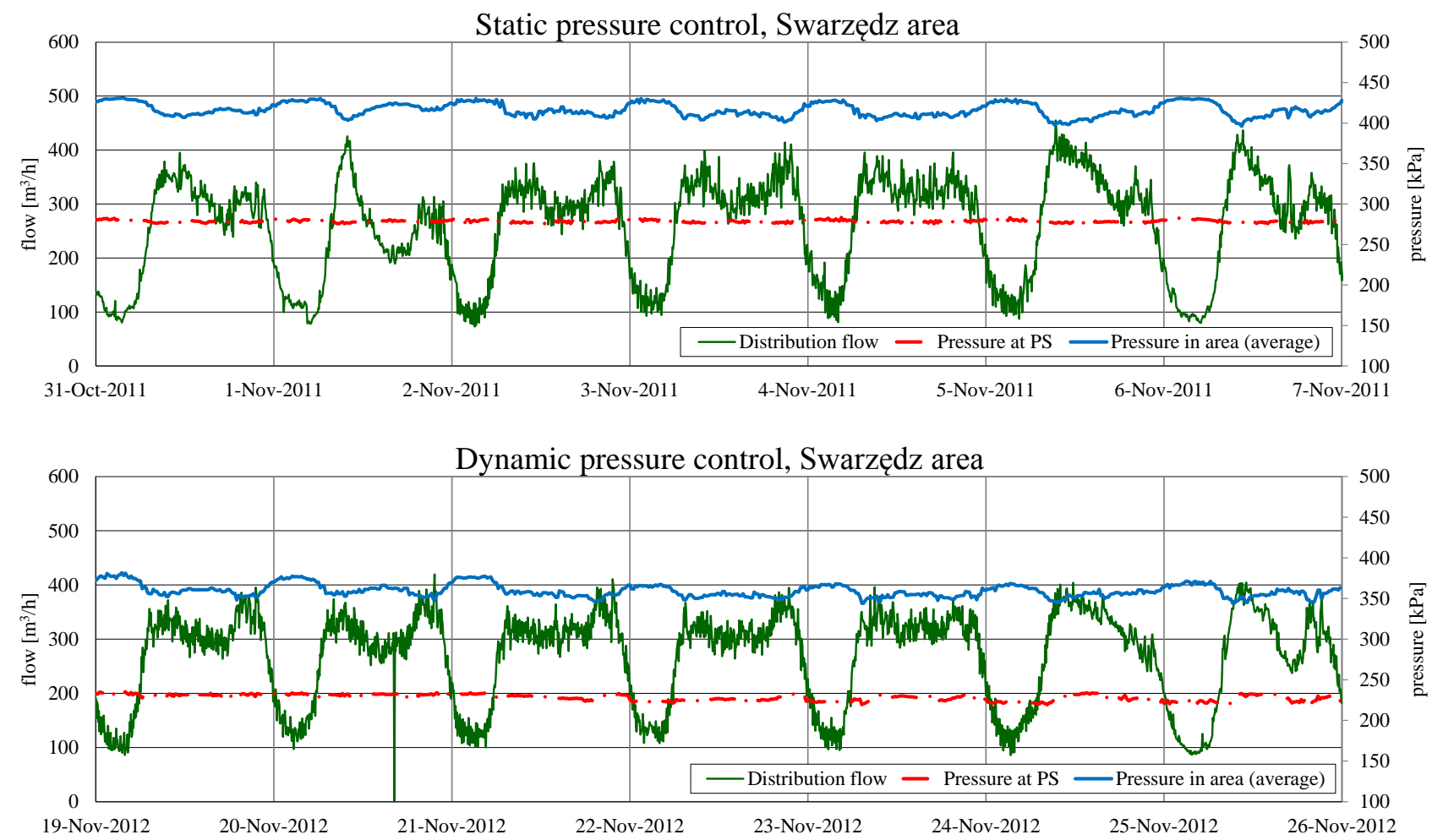

Figure 8: Difference between static (upper graph) and dynamic (lower graph) pressure control,

Swarzędz area

In the initial setup of the water supply system, a PRV was reducing the pressure to one of the two supply areas (Swarzędz area, see Figure 1). The pumps were operated at a fixed pressure (330 $\mathrm{kPa})$, and the fixed outlet PRV was set to reduce the pressure to the Swarzędz area to $280 \mathrm{kPa}$. The outlet pressure of the PRV was not constant, but was conversely related to the flow. The behaviour of the PRV is shown in Figure 9.

Figure 10 shows the outlet pressure at the clear water pumping station when it was controlled by the DPCM. The trend shows that the outlet pressure was lower during low flows and higher during high flows. This was caused by the fact that the pressure set-point for the pumping station was based on the (calculated) minimum pressure in the distribution network. Using the DPCM resulted, as expected, in a quadratic relation between the pressure and the flow, as can be seen in the lower graph of Figure 10. 

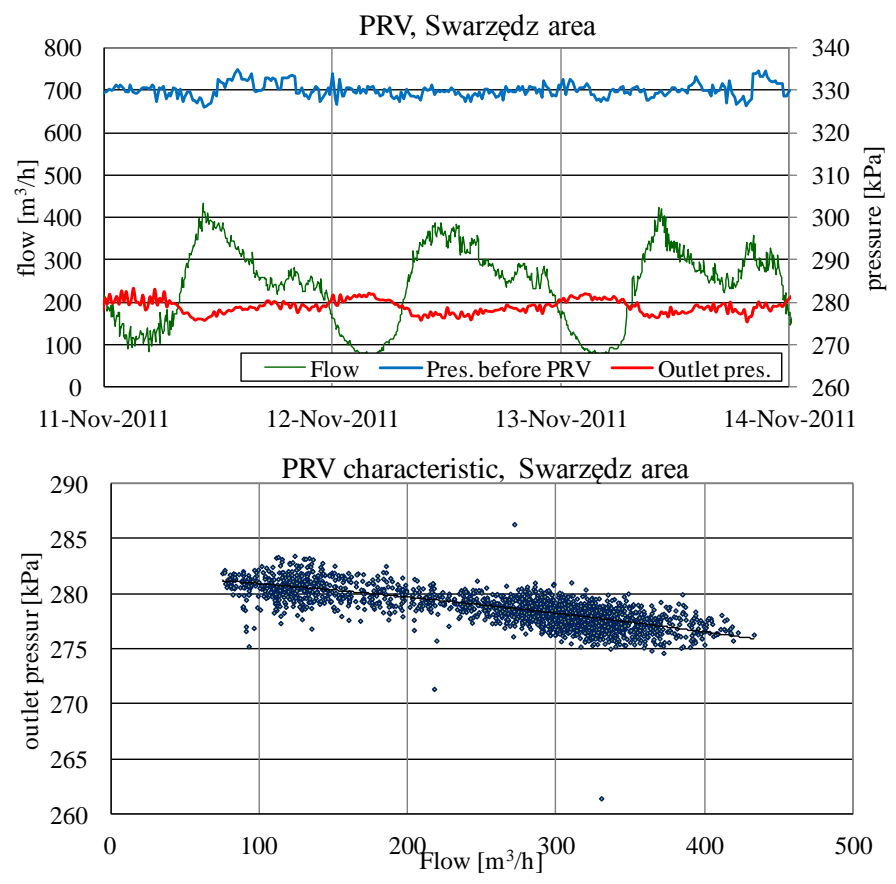

Figure 9: Outlet pressure in the water main to Swarzedz area with conventional pressure control including PRV
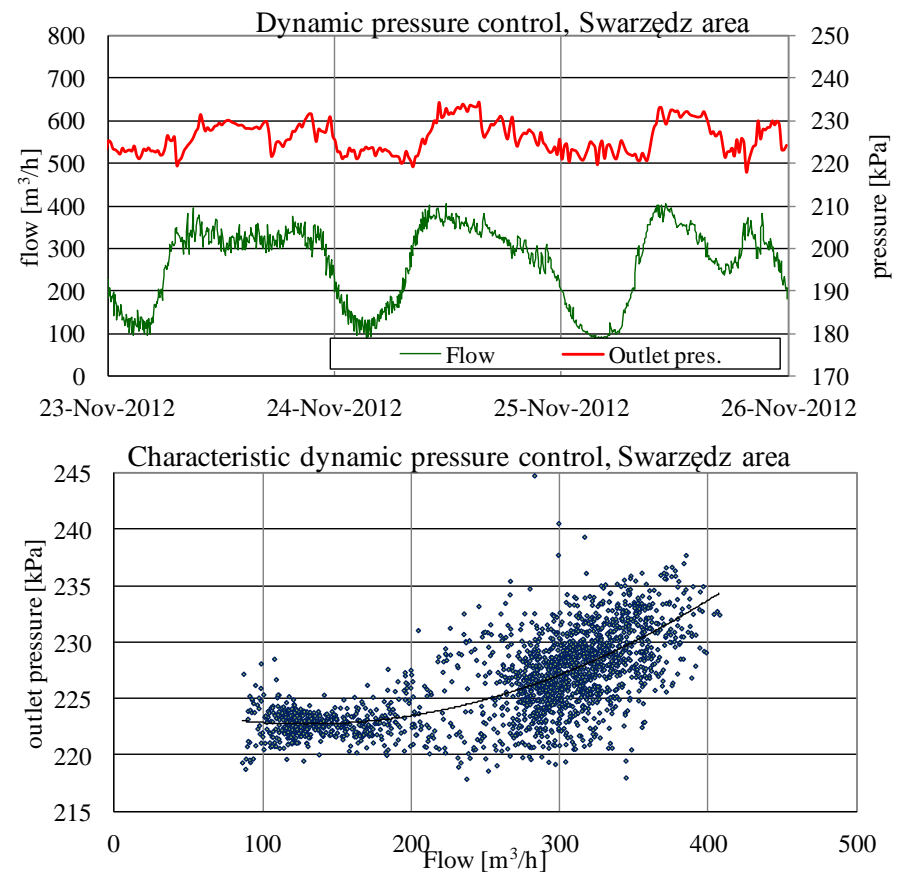

Figure 10: Outlet pressure in the water main to Swarzędz area with dynamic pressure control

The pump pressure of the clear water pumping station was $97 \mathrm{kPa}$ lower (29\%) when controlled by the DPCM, see Table 2. The average pressure in the distribution network was $100 \mathrm{kPa}(23 \%)$ lower in the Poznań area and $50 \mathrm{kPa}(12 \%)$ in the Swarzędz area. The difference in pressure in the 
Swarzędz area was smaller, because in the initial setup of the system the pressure was already reduced in this area with a PRV (reduction by $50 \mathrm{kPa}$ on average). The water flow to the Poznań area was in the period with dynamic pressure control $92 \mathrm{~m}^{3} / \mathrm{h}(43 \%)$ higher than in the period with static control. This increase was caused by a large industrial customer in the area, who increased its water consumption. The water flow to the Swarzędz area was almost equal in the two periods.

Table 2: Difference between static and dynamic pressure control

\begin{tabular}{|c|c|c|c|c|}
\hline & Level based & Model based & $\begin{array}{c}\text { Difference } \\
\text { pressure }\end{array}$ & $\begin{array}{c}\text { Difference } \\
\text { leakage }\end{array}$ \\
\hline Poznań area & & & & \\
\hline Flow $\left[\mathrm{m}^{3} / \mathrm{h}\right]$ & 214 & 306 & $+43 \%$ & \\
\hline Pumping station $[\mathrm{kPa}]$ & 330 & 233 & $-29 \%$ & \\
\hline $\mathrm{MP} 1[\mathrm{kPa}]$ & 395 & 298 & $-25 \%$ & \\
\hline $\mathrm{MP} 2[\mathrm{kPa}]$ & 475 & 373 & $-22 \%$ & \\
\hline MP3 $[\mathrm{kPa}]$ & 462 & 361 & $-22 \%$ & \\
\hline Area avg. [kPa] & 444 & 344 & $-23 \%$ & $-26 \%$ \\
\hline Swarzędz area & & & & \\
\hline Flow $\left[\mathrm{m}^{3} / \mathrm{h}\right]$ & 261 & 267 & $+2 \%$ & \\
\hline Outlet $[\mathrm{kPa}]$ & 279 & 233 & $-16 \%$ & \\
\hline $\mathrm{MP} 1[\mathrm{kPa}]$ & 470 & 422 & $-10 \%$ & \\
\hline $\mathrm{MP} 2[\mathrm{kPa}]$ & 516 & 452 & $-12 \%$ & \\
\hline MP3 $[\mathrm{kPa}]$ & 439 & 390 & $-11 \%$ & \\
\hline $\mathrm{MP} 4[\mathrm{kPa}]$ & 365 & 318 & $-13 \%$ & \\
\hline MP5 $[\mathrm{kPa}]$ & 329 & 281 & $-14 \%$ & \\
\hline MP6 $[\mathrm{kPa}]$ & 381 & 337 & $-12 \%$ & \\
\hline Area avg. [kPa] & 417 & 367 & $-12 \%$ & $-14 \%$ \\
\hline Total area avg. & 431 & 355 & $-17 \%$ & $-20 \%$ \\
\hline
\end{tabular}

The lower pressure in the area when controlled by the DPCM resulted in lower water losses in the distribution network. By applying equation (5), the background leakage was calculated to be $26 \%$ lower in the Poznań area, and 14\% lower in the Swarzędz area, resulting in 20\% lower in the entire

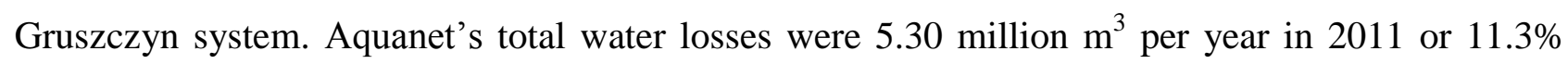
(Aquanet, 2012). With the above, we estimated the water losses in the Gruszczyn system in 2011 at $565,000 \mathrm{~m}^{3}$ per year and in 2012 at $450,000 \mathrm{~m}^{3}$ per year. 


\section{Reduction of energy consumption}

The reduction of energy consumption due to the implementation of advanced control software consists of three elements:

1. Savings due to production flow control.

2. Savings due to lower pump pressure of clear water pumps.

3. Saving due to reduced water losses.

Table 1 showed that the energy costs were $2.7 \%$ lower with model based production flow control. With a total annual production of WTP Gruszczyn of 5 million $\mathrm{m}^{3}$ per year, the implementation of model based production flow control led to a reduction in energy consumption of 43,500 $\mathrm{kWh}$ per year. The energy reduction due to lower pump pressure was calculated with equation (7). With a pumped volume $(V)$ of 5 million $\mathrm{m}^{3}$ per year, and a difference of the pump head $(d p)$ of $97 \mathrm{kPa}$, this results in a $d E_{\text {pump }}$ of $225,000 \mathrm{kWh}$ per year $(€ 13,550)$. The energy reduction due to reduced water losses was calculated with equation (6). Based on a reduced water loss of $113,500 \mathrm{~m}^{3}$ per year (see above), the reduction in energy consumption was calculated at $68,500 \mathrm{kWh}$ per year. The overall reduction of the energy consumption was $337,000 \mathrm{kWh}(€ 21,500)$ per year which corresponds to $11.5 \%$ of the total energy costs. The reduction of the pump pressure is the main contributor to the reduction of the overall energy consumption.

\section{Discussion}

\section{Increased water consumption}

Surprisingly, in the year with advanced control the water flows from the pumping station to the areas were higher than in the year with conventional control (Poznań area $+48 \%$; Swarzędz area $+2 \%$ ). A lower rather than a higher flow was expected, because the pressure in the areas was reduced significantly, and therefore significant reduction in background leakages were expected. The higher pump flows to the both areas can be explained by an increase of the water consumption. Especially, the higher water flow to the Poznan area can be linked to the increased water consumption of a large industrial customer in that area.

\section{Availability of pressure measurements}

An important difference between the periods of conventional control and advanced control, was the availability of pressure measurements in the distribution area. Initially, only two (unreliable) measurements were available. The operators had no confidence in the measurements and therefore 
chose a set-point based on their experience to operate the pumping station. The installation of new (more reliable) measurements showed that the pressure in the network was unnecessarily high. By using the new measurements, the advanced controller was able to reduce the pump pressure by $29 \%$. However, if only new pressure measurements were installed and no advanced controller, the operators would have reduced the pumping pressure as well based on the observation of unnecessarily high pressures in the network. In this case, the availability of reliable measurements was presumably more important than the introduction of an advanced controller. Still, the combination of installing new sensors and introducing advanced control is an important success factor to achieve efficiency improvements, because the sensors and models help to understand the possible improvements, and the advanced control helps to achieve the best operational results continuously.

\section{Static and flow modulated PRVs versus the DPCM}

The installed PRV to reduce the pressure in the Swarzędz area was a classic medium controlled valve. Prescott and Ulanicki (2003) used this type of valve to develop their dynamic model of PRVs. According to this model, this type of PRV is somewhat flow dependent: during low flows the outlet pressure is slightly higher than during high flows. This behaviour was also observed in the trends of flow and pressure to the Swarzędz area (Figure 9). However, this behaviour is undesirable. During low flows the dynamic head loss between the pumping station and the distribution area was lower, and therefore, a lower, instead of a higher, outlet pressure was desired.

The DPCM worked as a flow modulated PRV, which is also available as integrated medium controlled device, like the AQUAI-MOD ${ }^{\circledR}$ hydraulic controller (Abdelmeguid et al., 2011). Both the DPCM and medium controlled valves are able to reduce the outlet pressure during low flow, and increase the outlet pressure during higher flows. The advantage of the DPCM over a flow modulated PRV, is that the DPCM can be tuned easier, and that the DPCM adapts automatically to changing hydraulic or demand characteristics. A potential drawback of the DPCM is that it needs power and functioning communication infrastructure, which is not necessary for a medium controlled device. In the considered case study, this was not an issue because the pressure was controlled at the treatment facility which had a permanent and failsafe power supply and communication infrastructure. 


\section{Reduction of pipe breaks}

Girard and Stewart (2007) and Gomes et al. (2011) showed that a reduction in the pressure in a water supply area also leads to a reduction in the number of main breaks and service breaks. Based on the lower pressure in the area when controlled by the DPCM, a lower number of breaks may be expected in this case study as well. However, the number of breaks in the concerning areas were not registered separately, making it impossible to confirm the expected reduction in breaks.

\section{Conclusion}

Many water supply systems are still controlled by basic conventional control loops. Though this control is reliable and easy to understand, it is inefficient with respect to energy consumption and leakage. More advanced controllers can help to improve the efficiency of the water supply systems. The advanced production flow control resulted in an $80 \%$ reduction of the production flow variation, and the dynamic pressure control resulted in a $29 \%$ reduction of the pump pressure and a $20 \%$ reduction of the background leakage compared to conventional control. We estimated the reductions in the operational costs due to the reduced energy consumption $(337,000 \mathrm{kWh}$ per year, or $€ 21,500$ per year) and the $20 \%$ lower water losses. Based on these results, we conclude that extra information from the distribution network (nine reliable pressure measuring points) in combination with advanced control software led to a more efficient water supply system.

\section{Acknowledgements}

The project is financially supported by the Dutch government through the "Partners for Water" programme. The project was implemented by Royal HaskoningDHV the Netherlands, supported by water utility Oasen (the Netherlands), water utility Aquanet (Poland) and Royal HaskoningDHV Poland.

\section{References}

Abdelmeguid, H., Skworcow, P., and Ulanicki, B. (2011). "Mathematical modelling of a hydraulic controller for PRV flow modulation". Journal of Hydroinformatics. 13 (3): 374-389.

Aquanet. 2012. Annual report 2011.

Araujo, L.S., Ramos, H., and Coelho, S.T. (2006). "Pressure control for leakage minimisation in water distribution systems management". Water Resources Management. 20 (1): 133-149.

Bakker, M., Van Schagen, K., and Timmer, J. (2003). "Flow control by prediction of water demand". Journal of Water Supply: Research and Technology - AQUA. 52 (6): 417-424.

Bakker, M., Vreeburg, J.H.G., Palmen, L.J., Sperber, V., Bakker, G., and Rietveld, L.C. (2013a). "Better water quality and higher energy efficiency by using model predictive flow control at water supply systems". Journal of Water Supply: Research and Technology - AQUA. 62 (1): 
$1-13$.

Bakker, M., Vreeburg, J.H.G., van Schagen, K.M., and Rietveld, L.C. (2013b). "A fully adaptive forecasting model for short-term drinking water demand". Environmental Modelling and Software. 48 (1): 141-151.

Bunn, S. (2007). "Closing the loop in water supply optimisation", Proceedings, Coventry.

Bunn, S.M., and Reynolds, L. (2009). "The energy-efficiency benefits of pumpscheduling optimization for potable water supplies". IBM Journal of Research and Development. 53 (3).

Colombo, A.F., and Karney, B.W. (2005). "Impacts of leaks on energy consumption in pumped systems with storage". Journal of Water Resources Planning and Management. 131 (2): 146-155.

Girard, M., and Stewart, R.A. (2007). "Implementation of pressure and leakage management strategies on the gold coast, Australia: Case study". Journal of Water Resources Planning and Management. 133 (3): 210-217.

Gomes, R., Marques, A.S., and Sousa, J. (2011). "Estimation of the benefits yielded by pressure management in water distribution systems". Urban Water Journal. 8 (2): 65-77.

Hydratek. 2013. Toward Municipal Sector Conservation: A Pump Efficiency Assessment and Awareness Pilot Study. Toronto, Ontario, Canada.

May, J. (1994). "Pressure dependent leakage". World Water \& Environmental Engineer. 17 (8): 10.

Prescott, S.L., and Ulanicki, B. (2003). "Dynamic modeling of pressure reducing valves". Journal of Hydraulic Engineering. 129 (10): 804-812.

PWN. (2006). "Automated Water System Control". Control Engineering. 53 (10).

Ulanicki, B., Bounds, P.L.M., Rance, J.P., and Reynolds, L. (2000). "Open and closed loop pressure control for leakage reduction". Urban Water. 2 (2): 105-114.

Vairavamoorthy, K., and Lumbers, J. (1998). "Leakage reduction in water distribution systems: Optimal valve control". Journal of Hydraulic Engineering. 124 (11): 1146-1154.

Worm, G.I.M., van der Helm, A.W.C., Lapikas, T., van Schagen, K.M., and Rietveld, L.C. (2010). "Integration of models, data management, interfaces and training support in a drinking water treatment plant simulator". Environmental Modelling and Software. 25 (5): 677-683. 